Doi: $10.15863 / \mathrm{TAS}$

\section{International Scientific Journal}

\section{Theoretical \& Applied Science}

p-ISSN: 2308-4944 (print) e-ISSN: 2409-0085 (online)

Year: $2015 \quad$ Issue: 02 Volume: 22

Published: 28.02.2015 http://www.T-Science.org
Gulzhan Kadirhanovna Rahimova

candidate of physico-mathematical Sciences, senior lecturer, Taraz state University named after

M.H. Dulati, Kazakhstan rahimovagk@mail.ru

Ainur Tursynhanovna Tolkynbayeva senior lecturer

Taraz state University named after M.H. Dulati,

Kazakhstan

Bereke Mukasheva

student

Taraz state University named after M.H. Dulati,

Kazakhstan

\title{
SMOOTHNESS AND EXISTENCE OF SOLUTIONS OF SOME NONLINEAR DEGENERATE EQUATIONS
}

\begin{abstract}
In this paper there is investigated the existence, smoothness and approximation properties of a solutions of a class of degenerate loaded equations of non-classical type.

Key words: non-classical equation, nonlinear equation, boundary value problem, the smoothness.

Language: Russian

Citation: Rahimova GK, Tolkynbayeva AT, Mukasheva B (2015) SMOOTHNESS AND EXISTENCE OF SOLUTIONS OF SOME NONLINEAR DEGENERATE EQUATIONS. ISJ Theoretical \& Applied Science 02 (22): 82-87. doi: http://dx.doi.org/10.15863/TAS.2015.02.22.14
\end{abstract}

\section{УДК 517.946}

\section{О ГЛАДКОСТИ И СУЩЕСТВОВАНИИ РЕШЕНИЙ НЕКОТОРЫХ НЕЛИНЕЙНЫХ ВЫРОЖДАЮЩИХСЯ УРАВНЕНИЙ}

Аннотация: В статье исследованы существование, гладкость и аппроксимативные свойства решений одного класса вырождаюшихся нелинейных уравнений неклассического типа.

Ключевые слова: неклассическое уравнение, нелинейное уравнение, краевая задача, гладкость.

1. Введение и формулировка основных условий.

В течений нескольких десятилетий общая теория краевых задач для вырождающихся эллиптических уравнений интенсивно изучается в связи с их большим значением для приложений (например, в газовой динамике). Несмотря на это, ещё нет окончательного ответа на вопросы, рассматриваемые нами: теоремы о гладкости решений, оценки промежуточных производных решений с различными весовыми функциями.
В настоящей работе получены следующие результаты: предельная гладкость решений одного класса вырождающихся уравнений в случае неограниченной области; необходимые и достаточные условия для оценки производных решений с различными весовыми функциями. Эти результаты дополняют работы С.М.Никольского [1], П.И.Лизоркина [2], М.И.Вишика [3], Т.Ш.Кальменова, М.Отелбаева [4], М.Б.Муратбекова [5].

В $L_{2}(\Omega)$ рассматривается следующая задача:

$$
\begin{gathered}
L u=-\frac{\partial^{2} u}{\partial y^{2}}+\sum_{k=0}^{s}(-1)^{k} R_{k}(x, y, u) \frac{\partial^{2 k+1} u}{\partial x^{2 k+1}}+\sum_{k=0}^{m}(-1)^{k} B_{k}(x, y, u) \frac{\partial^{2 k} u}{\partial x^{2 k}}=f \in L_{2}(\Omega), \\
\left.\frac{\partial^{i} u}{\partial x^{i}}\right|_{x=0}=\left.\frac{\partial^{i} u}{\partial x^{i}}\right|_{x=2 \pi}, \quad i=0,1,2, \ldots, 2 s, \quad s \geq m,
\end{gathered}
$$




$$
u(x, 0)=u(x, 1)=0,
$$

где $f \in L_{2}(\Omega), \quad \Omega=\{(x, y): 0<x<2 \pi, \quad 0<y<1\}$.

В дальнейшем считаем, что функции

$$
R_{k}(x, y, z) \quad(k=0,1, \ldots, s), \quad B_{k}(x, y, z) \quad(k=0,1, \ldots, m)
$$

кусочно-непрерывны и ограниченны по заданным аргументам и удовлетворяют условиям:

$$
\text { a) } C^{-1} \varphi_{k}(y) \leq R_{k}(x, y, z) \leq C \varphi_{k}(y), x \in[0,2 \pi], \quad y \in[0,1],|z| \in[0, A],
$$

A- любое фиксированное число ;

б) $C^{-1} \psi_{k}(y) \leq B_{k}(x, y, z) \leq C \psi_{k}(y), \quad x \in[0,2 \pi], \quad y \in[0,1],|z| \in[0, A]$,

где функиии $\varphi_{k} \quad u \quad \psi_{k}$ удовлетворяют

соответственно условиям в)-2).

в) $R_{k}(y) \geq 0(k=1,2, \ldots, s), B_{k}(y) \geq 0(k=2, \ldots, m), R_{0}(y) \geq \delta_{0}>0, B_{0}(y) u B_{1}(y) \geq \delta>0$.

2) $\lim _{y \rightarrow 0} \frac{R_{k}(2 y)}{R_{k}(y)}<\infty \quad(k=1,2, \ldots, s) ; \quad \lim _{y \rightarrow 0} \frac{B_{k}(2 y)}{B_{k}(y)}<\infty(k=1,2, \ldots, m)$.

Теорема 1. Пусть выполнены условия: $а$ )-б)

Тогда существует решение задачи (1)-(3), и для него справедлива оценка:

$$
\|u\|_{C(\bar{\Omega})} \leq C_{0}\|f\|_{2}, \quad\left(\|u\|_{W_{2}^{2}(\Omega)} \leq C\|f\|_{2}\right),
$$

где С и $C_{0}>0$ - постоянные числа.

\section{2. Вспомогательные оценки}

Прежде, чем доказать эту теорему, приводим несколько вспомогательных предложений.

Рассмотрим задачу:

$$
\begin{gathered}
L_{v} u=-\frac{\partial^{2} u}{\partial y^{2}}+\sum_{k=0}^{s}(-1)^{k} R_{k}(x, y, v) \frac{\partial^{2 k+1} u}{\partial x^{2 k+1}}+\sum_{k=0}^{m}(-1)^{k} B_{k}(x, y, v) \frac{\partial^{2 k} u}{\partial x^{2 k}}=f \in L_{2}(\Omega), \\
\left.\frac{\partial^{i} u}{\partial x^{i}}\right|_{x=0}=\left.\frac{\partial^{i} u}{\partial x^{i}}\right|_{x=2 \pi}, \quad i=0,1,2, \ldots, 2 s, \quad s \geq m, \\
u(x, 0)=u(x, 1)=0,
\end{gathered}
$$

Лемма 2.1 Пусть $v \in C(\bar{\Omega})$ и выполнено условие $а$ )-б). Тогда для любой правой части $f$ из $L_{2}(\Omega)$ существует, притом единственное решение задачи (5)-(7) и для него справедлива оиенка:

$$
\|u(x, y)\|_{C(\bar{\Omega})} \leq C\|f\|_{2}, \quad\left(\|u\|_{W_{2}^{2}(\Omega)} \leq C_{0}\|f\|_{2}\right),
$$

где $C_{0}, C>0$ не зависит от $u, v$.

Доказательство. Положим

$$
\widetilde{R}_{k}(y)=R_{k}(x, y, v),(k=0,1, \ldots, s), \quad \tilde{B}_{k}(y)=B_{k}(x, y, v),(k=0,1, \ldots, m) .
$$


Тогда (5)-(7) сводится к задаче (1)-(3), где функции $\quad R_{k}(y), B_{k}(y) \quad$ заменены, соответственно, на $\tilde{R}_{k}(y), \tilde{B}_{k}(y)$. При этом согласно условию $a)-\sigma)$ для $\tilde{R}_{k}(y), \tilde{B}_{k}(y)$ выполняются все условия теоремы [6], откуда вытекает утверждение доказываемой леммы. Таким образом, задача (5)-(7) имеет единственное решение $u=L_{v}^{-1} f$ удовлетворяющее оценку (8). Очевидно, если $v \in C(\bar{\Omega}), \quad$ то $u=L_{v}^{-1} f \in \tilde{N}(\bar{\Omega})$. Более того, поскольку $u=L_{v}^{-1} f-$ решение задачи (5)-(7), для произвольной функции $v \in C(\bar{\Omega})$ имеем $L_{v}^{-1} f \in D(L)$. Поэтому, существование решения краевой задачи (5)-(7) эквивалентно существованию неподвижной точки оператор $L_{v}^{-1}$ в пространстве $C(\bar{\Omega})$ т.е. существование функции $u \in C(\bar{\Omega})$ такой, что $u=L_{u}^{-1} f$. При этом $u \in D(L)$, поскольку $L_{u}^{-1} f \in D(L)$.

Следовательно, задача (5)-(7) имеет неподвижную точку. С этой целью применяем известный принцип Шаудера.

$$
\text { Пусть } \quad \bar{S}=\left\{v \in C(\bar{\Omega}):\|v\|_{C(\bar{\Omega})} \leq A\right\} \quad-
$$

шар в пространстве $C(\bar{\Omega})$ и А- произвольное положительное число.

Лемма 2.2 Пусть выполнено условие а) -б) . Тогда оператор $L_{v}^{-1}$ отображает множество $\bar{S}$ в себя.

Доказательство. Доказательство леммы следует из теоремы 2 [6] и леммы 2.1, если в качестве А взять число $C\|f\|_{2}$ из оценки (8). Пусть $M=\left\{u \in C(\bar{\Omega}): u=L_{v}^{-1} f, v \in \bar{S}\right\}$ - прообраз шара $\bar{S}$.

Лемма 2.3 Пусть выполнено условие а)-б)

. Тогда оператор $L_{v}^{-1}$ непрерывен.

Доказательство. Пусть последовательность $\left\{v_{n}\right\}_{n=1}^{\infty}$ и элемент $v$ из множества $\bar{S}$, такие, что $v_{n} \rightarrow v$ в норме пространства $C(\bar{\Omega})$.

Положим $L_{v} u=f$ и решение, если оператор $L_{v}^{-1}$ имеет

$$
\begin{aligned}
& L_{v_{n}} u=-\frac{\partial^{2} u_{n}}{\partial y^{2}}+\sum_{k=0}^{s}(-1)^{k} R_{k}\left(x, y, v_{n}\right) \frac{\partial^{2 k+1} u_{n}}{\partial x^{2 k+1}}+ \\
& +\sum_{k=0}^{m}(-1)^{k} B_{k}\left(x, y, v_{n}\right) \frac{\partial^{2 k} u_{n}}{\partial x^{2 k}}=f,
\end{aligned}
$$

где $f(x)$ - фиксированный элемент в $L_{2}(\Omega)$.

Тогда

$$
\begin{aligned}
&-\left(\frac{\partial^{2} u_{n}}{\partial y^{2}}-\frac{\partial^{2} u}{\partial y^{2}}\right)+\sum_{k=0}^{s}(-1)^{k} R_{k}\left(x, y, v_{n}\right) \frac{\partial^{2 k+1} u_{n}}{\partial x^{2 k+1}}+ \\
&+\sum_{k=0}^{m}(-1)^{k} B_{k}\left(x, y, v_{n}\right) \frac{\partial^{2 k} u_{n}}{\partial x^{2 k}}-\sum_{k=0}^{s}(-1)^{k} R_{k}(x, y, v) \frac{\partial^{2 k+1} u}{\partial x^{2 k+1}}- \\
&-\sum_{k=0}^{m}(-1)^{k} B_{k}(x, y, v) \frac{\partial^{2 k} u}{\partial x^{2 k}}=0,
\end{aligned}
$$

или 


$$
\begin{aligned}
& -\left(\frac{\partial^{2} u_{n}}{\partial y^{2}}-\frac{\partial^{2} u}{\partial y^{2}}\right)+\sum_{k=0}^{s}(-1)^{k} R_{k}\left(x, y, v_{n}\right)\left(\frac{\partial^{2 k+1} u_{n}}{\partial x^{2 k+1}}-\frac{\partial^{2 k+1} u}{\partial x^{2 k+1}}\right)+ \\
& \quad+\sum_{k=0}^{m}(-1)^{k} B_{k}\left(x, y, v_{n}\right)\left(\frac{\partial^{2 k} u_{n}}{\partial x^{2 k}}-\frac{\partial^{2 k} u}{\partial x^{2 k}}\right)= \\
& =\left(\sum_{k=0}^{s}(-1)^{k}\left[R_{k}(x, y, v)-R_{k}\left(x, y, v_{n}\right)\right]\right) \frac{\partial^{2 k+1} u}{\partial x^{2 k+1}}+ \\
& +\left(\sum_{k=0}^{m}(-1)^{k}\left[B_{k}(x, y, v)-B_{k}\left(x, y, v_{n}\right)\right]\right) \frac{\partial^{2 k} u}{\partial x^{2 k}} .
\end{aligned}
$$

Левая часть последнего равенства имеет вид:

$$
\begin{aligned}
L_{v_{n}}\left(u_{n}-u\right) & =-\left(\frac{\partial^{2} u_{n}}{\partial y^{2}}-\frac{\partial^{2} u}{\partial y^{2}}\right)+\sum_{k=0}^{s}(-1)^{k} R_{k}\left(x, y, v_{n}\right)\left(\frac{\partial^{2 k+1} u_{n}}{\partial x^{2 k+1}}-\frac{\partial^{2 k+1} u}{\partial x^{2 k+1}}\right)+ \\
& +\sum_{k=0}^{m}(-1)^{k} B_{k}\left(x, y, v_{n}\right)\left(\frac{\partial^{2 k} u_{n}}{\partial x^{2 k}}-\frac{\partial^{2 k} u}{\partial x^{2 k}}\right) .
\end{aligned}
$$

Отсюда и из (9) находим:

$$
\begin{aligned}
L_{v_{n}}\left(u_{n}-u\right) & =\left(\sum_{k=0}^{s}(-1)^{k}\left[R_{k}(x, y, v)-R_{k}\left(x, y, v_{n}\right)\right]\right) \frac{\partial^{2 k+1} u}{\partial x^{2 k+1}}+ \\
& +\left(\sum_{k=0}^{m}(-1)^{k}\left[B_{k}(x, y, v)-B_{k}\left(x, y, v_{n}\right)\right]\right) \frac{\partial^{2 k} u}{\partial x^{2 k}}
\end{aligned}
$$

По предположению, коэффициенты

оператора $L_{v_{n}}$ удовлетворяют условиям

теоремы 1 [6-9], следовательно существует обратный оператор

$$
\begin{aligned}
u_{n}-u= & L_{v_{n}}^{-1}\left[\left(\sum_{k=0}^{s}(-1)^{k}\left[R_{k}(x, y, v)-R_{k}\left(x, y, v_{n}\right)\right]\right) \frac{\partial^{2 k+1} u}{\partial x^{2 k+1}}+\right. \\
& \left.+\left(\sum_{k=0}^{m}(-1)^{k}\left[B_{k}(x, y, v)-B_{k}\left(x, y, v_{n}\right)\right]\right) \frac{\partial^{2 k} u}{\partial x^{2 k}}\right]
\end{aligned}
$$

Отсюда

$$
\begin{gathered}
\lim _{n \rightarrow \infty}\left\|u_{n}-u\right\|_{C(\bar{\Omega})}=\lim _{n \rightarrow \infty} \| L_{v_{n}}^{-1}\left[\left(\sum_{k=0}^{s}(-1)^{k}\left[R_{k}(x, y, v)-R_{k}\left(x, y, v_{n}\right)\right]\right) \frac{\partial^{2 k+1} u}{\partial x^{2 k+1}}+\right. \\
\left.+\left(\sum_{k=0}^{m}(-1)^{k}\left[B_{k}(x, y, v)-B_{k}\left(x, y, v_{n}\right)\right]\right) \frac{\partial^{2 k} u}{\partial x^{2 k}}\right] \|_{C(\bar{\Omega})} .
\end{gathered}
$$

Пользуясь теоремами 1 и 2 [6-9] имеем: 


$$
\begin{gathered}
\lim _{n \rightarrow \infty}\left\|u_{n}-u\right\|_{C(\bar{\Omega})} \leq \lim _{n \rightarrow \infty}\left\|L_{u_{n}}^{-1}\right\|_{C(\bar{\Omega})} \\
{\left[\sum_{k=0}^{s}\left\|(-1)^{k}\left[R_{k}(x, y, v)-R_{k}\left(x, y, v_{n}\right)\right] \frac{\partial^{2 k+1} u}{\partial x^{2 k+1}}\right\|_{2}+\right.} \\
\left.+\left[\sum_{k=0}^{m}\left\|(-1)^{k}\left[B_{k}(x, y, v)-B_{k}\left(x, y, v_{n}\right)\right] \frac{\partial^{2 k} u}{\partial x^{2 k}}\right\|_{2}\right]\right\} .
\end{gathered}
$$

Из теоремы 1 [6] также следует, что

$$
\left\|L_{v_{n}}^{-1} f\right\|_{C(\bar{\Omega}) \rightarrow C(\bar{\Omega})} \leq C\|f\|_{2},
$$

для всех $\left\{u_{n}\right\}_{n=1}^{\infty}$ и $v$. А из (8) получим оценки:

$$
\begin{aligned}
& \sum_{k=0}^{s}\left\|(-1)^{k}\left[R_{k}(x, y, v)-R_{k}\left(x, y, v_{n}\right)\right] \frac{\partial^{2 k+1} u}{\partial x^{2 k+1}}\right\|_{2} \rightarrow 0, \text { при } n \rightarrow \infty, \\
& \sum_{k=0}^{m}\left\|(-1)^{k}\left[B_{k}(x, y, v)-B_{k}\left(x, y, v_{n}\right)\right] \frac{\partial^{2 k} u}{\partial x^{2 k}}\right\|_{2} \rightarrow 0, \text { при } n \rightarrow \infty,
\end{aligned}
$$

Учитывая оценки (11)-(13), из неравенства (10) имеем:

$$
\lim _{n \rightarrow \infty}\left\|u_{n}-u\right\|_{C(\bar{\Omega})} \rightarrow \infty .
$$

Здесь мы воспользовались тем, что $\left\|v_{n}-v\right\|_{C(\bar{\Omega})} \rightarrow 0$ при $n \rightarrow \infty$.

Неравенство (14) доказывает лемму.

Доказательство теоремы 1. Согласно лемме 2.3 оператор $L_{v}^{-1}$ вполне непрерывен и переводит шар $\bar{S}$ в себя. Тогда согласно принципу Шаудера оператор $L_{v}^{-1}$ при фиксированном $f(x) \in L_{2}(\Omega) \quad$ имеет неподвижную точку в $\bar{S}$. Это означает, что задачи (1)-(3) для любой правой части $f(x) \in L_{2}(\Omega)$ имеет решение $u(x, y)$, принадлежащее шару $\bar{S}$, причем верна оценка:

$$
\begin{aligned}
\|u\|_{C(\bar{\Omega})} & \leq C_{0}\|f\|_{2}, \\
\|u\|_{W_{2}^{2}(\Omega)} & \leq C\|f\|_{2},
\end{aligned}
$$

Теорема доказана.

\section{References:}

1. Nikol'skiy SM (1979) Variatsionnaya problema dlya uravneniya ellipticheskogo tipa vyrozhdeniem na granitse. Trudy MI AN SSSR, 1979, t.150, pp.212-238.

2. Lizorkin PI, Nikol'skiy SM (1981) Ellipticheskie uravneniya $\mathrm{s}$ vyrozhdeniem. Differentsial'nye svoystva resheniy. Dokl. AN SSSR, 1981,t.257, №1, pp.42-45.
3. Vishik MI, Grushin VV (1969) Kraevye zadachi dlya ellipticheskikh uravneniy vyrozhdayushchikhsya na granitse oblasti. Matematicheskiy sbornik, 1969, t.80, №4, pp.455-491.

4. Kal'menov TS, Otelbaev M (1977) O gladkosti resheniy odnogo klassa vyrozhdayushchikhsya ellipticheskikh uravneniy. Differentsial'nye uravneniya, 1977, t.13, №7, pp.1244-1255. 
5. Muratbekov MB (1981) Koertsitivnye otsenki dlya odnogo differentsial'nogo operatora vysokogo poryadka. Differentsial'nye uravneniya, 1981, t.17, №5, pp.893-901.

6. Rakhimova GK (2006) Apriornye otsenki resheniy neklasicheskogo uravneniya tret'ego poryadka $\mathrm{v}$ neogranichennoy oblasti . Tezisy dokladov mezhdunarodnoy 11-oy mezhvuzovskoy konferentsii po matematike $\mathrm{i}$ mekhanike. - Astana, 2006.

7. Muratbekov MB (1981) O gladkosti resheniy odnogo klassa neravnomerno vyrozhdayushchikhsya ellipticheskikh uravneniy. Izvestiya AN Kaz SSR. Ser.fiz-mat. 1981. №5. pp.71-73.

8. Muratbekov MB (1981) O gladkosti resheniy odnogo klassa neravnomerno vyrozhdayushchikhsya ellipticheskikh uravneniy vysokogo poryadka. Korrektnye kraevye zadachi dlya neklassicheskikh uravneniy matematicheskoy fiziki. Novosibirsk. 1981. pp.144-146.

9. Muratbekov MB (1982) O gladkosti resheniy vyrozhdayushchikhsya ellipticheskikh uravneniy i odnomernogo nelineynogo statsionarnogo uravneniya Shredingera. Avtoreferat diss. kand. Almaty. 1982. pp.16.
10. Muratbekov MB, Muratbekov MM, Ospanov KN (2006) Ob approksimativnykh svoystvakh resheniya nelineynogo uravneniya smeshannogo tipa. Fundamental'naya i prikladnaya matematika. MGU. 2006. T.12. №5. pp.95-107.

11. Muratbekov MB, Muratbekov MM (2007) Otsenki spektra odnogo klassa operatorov smeshannogo tipa. Differentsial'nye uravneniya. RAN. 2007. T.43. №1. pp.135-138.

12. Mikhaylov VP (1976) Differentsial'nye uravneniya $\mathrm{V}$ chastnykh proizvodnykh. Moscow. Nauka. 1976.

13. Muratbekov MB (1991) Razdelimost' i otsenki poperechnikov mnozhestv, svyazannykh s oblast'yu opredeleniya nelineynogo operatora Shredingera. Differentsial'nye uravneniya. 1991. T.27. №6. pp. 1034 -1042.

14. Mynbaev KT, Otelbaev M (1988) Vesovye funktsional'nye prostranstva i spektr differentsial'nykh operatorov. Differentsial'nye uravneniya. Moscow. Nauka. 1988. pp. 286.

15. Muratbekov MB (2006) Razdelimost' i spektr differentsial'nykh operatorov smeshannogo tipa. -Taraz: -2006. -pp.163. 